OPEN ACCESS

Edited by: Susanne Röhr University Hospital Leipzig, Germany

Reviewed by:

Filip Morys,

McGill University, Canada

Rachel G. Zsido,

Max Planck Institute for Human Cognitive and Brain Sciences,

Germany

${ }^{*}$ Correspondence:

Changsoo Kim

preman@yuhs.ac

Young Noh

ynoh@gilhospital.com

Received: 13 April 2021

Accepted: 02 June 2021

Published: 23 June 2021

Citation:

Cho J, Seo S, Kim W-R, Kim C

and Noh Y (2021) Association Between Visceral Fat and Brain Cortical Thickness in the Elderly:

A Neuroimaging Study.

Front. Aging Neurosci. 13:694629.

doi: 10.3389/fnagi.2021.694629

\section{Association Between Visceral Fat and Brain Cortical Thickness in the Elderly: A Neuroimaging Study}

\author{
Jaelim Cho ${ }^{1,2}$, Seongho Seo ${ }^{3,4}$, Woo-Ram Kim ${ }^{5}$, Changsoo Kim ${ }^{1,2,6 *}$ and Young Noh ${ }^{7,8 *}$ \\ ${ }^{1}$ Institute for Environmental Research, Yonsei University College of Medicine, Seoul, South Korea, ${ }^{2}$ Institute of Human \\ Complexity and Systems Science, Yonsei University, Incheon, South Korea, ${ }^{3}$ Department of Electronic Engineering, Pai Chai \\ University, Daejeon, South Korea, ${ }^{4}$ Department of Neuroscience, College of Medicine, Gachon University, Incheon, \\ South Korea, ${ }^{5}$ Neuroscience Research Institute, Gachon University, Incheon, South Korea, ${ }^{6}$ Department of Preventive \\ Medicine, Yonsei University College of Medicine, Seoul, South Korea, ${ }^{7}$ Department of Neurology, Gil Medical Center, \\ Gachon University College of Medicine, Incheon, South Korea, ${ }^{8}$ Department of Health Science and Technology, GAlHST, \\ Gachon University, Incheon, South Korea
}

Background: Despite emerging evidence suggesting that visceral fat may play a major role in obesity-induced neurodegeneration, little evidence exists on the association between visceral fat and brain cortical thickness in the elderly.

Purpose: We aimed to examine the association between abdominal fat and brain cortical thickness in a Korean elderly population.

Methods: This cross-sectional study included elderly individuals without dementia $(n=316)$. Areas of visceral fat and subcutaneous fat $\left(\mathrm{cm}^{2}\right)$ were estimated from computed tomography scans. Regional cortical thicknesses $(\mathrm{mm})$ were obtained by analyzing brain magnetic resonance images. Given the inverted U-shaped relationship between visceral fat area and global cortical thickness (examined using a generalized additive model), visceral fat area was categorized into quintiles, with the middle quintile being the reference group. A generalized linear model was built to explore brain regions associated with visceral fat. The same approach was used for subcutaneous fat.

Results: The mean (standard deviation) age was 67.6 (5.0) years. The highest quintile (vs. the middle quintile) group of visceral fat area had reduced cortical thicknesses in the global $[\beta=-0.04 \mathrm{~mm}$, standard error $(\mathrm{SE})=0.02 \mathrm{~mm}, p=0.004]$, parietal $(\beta=-$ $0.04 \mathrm{~mm}, S E=0.02 \mathrm{~mm}, p=0.01)$, temporal $(\beta=-0.05 \mathrm{~mm}, S E=0.02 \mathrm{~mm}, p=0.002)$, cingulate $(\beta=-0.06 \mathrm{~mm}, S E=0.02 \mathrm{~mm}, \beta=0.01)$, and insula lobes $(\beta=-0.06 \mathrm{~mm}$, $S E=0.03 \mathrm{~mm}, p=0.02$ ). None of the regional cortical thicknesses significantly differed between the highest and the middle quintile groups of subcutaneous fat area.

Conclusion: The findings suggest that a high level of visceral fat, but not subcutaneous fat, is associated with a reduced cortical thickness in the elderly.

Keywords: abdominal fat, visceral fat, neuroimaging, cortical thickness, MRI 


\section{INTRODUCTION}

Obesity is a well-known risk factor for cardiovascular diseases, type 2 diabetes, and cancer (Bogers et al., 2007; Renehan et al., 2008; Bell et al., 2014). It has also been suggested that obesity is an independent risk factor for Alzheimer's disease and vascular dementia (Beydoun et al., 2008). To elucidate the effect of obesity on the brain in cognitively healthy individuals, a number of neuroimaging studies have investigated the association between obesity (including central obesity) and brain structure on magnetic resonance imaging (MRI) (Gunstad et al., 2008; Taki et al., 2008; Raji et al., 2010; Yokum et al., 2012; Kurth et al., 2013; Kim et al., 2015; Medic et al., 2016; Dekkers et al., 2019; Hamer and Batty, 2019; Morys et al., 2021). A largescale study of the United Kingdom Biobank $(n=9,652)$ showed that three obesity indices [body mass index (BMI), waist-tohip ratio (WHR), and total fat mass from body impedance] were significantly associated with a reduction in global gray matter volume (Hamer and Batty, 2019). Another study of the United Kingdom Biobank $(n=12,087)$ reported that the association between total fat mass from body impedance and global gray matter volume was significant only in men (Dekkers et al., 2019). Some of the neuroimaging studies have measured cortical thickness (Kim et al., 2015; Medic et al., 2016; Morys et al., 2021), a more sensitive indicator of gray matter changes than cortical volume (Burggren et al., 2008; Thambisetty et al., 2010). Kim et al. (2015) demonstrated inverse associations between total fat percentage from body impedance and WHR with region-ofinterest (ROI)-based global and frontal thicknesses only in men. Medic et al. (2016) found several focal regions in the frontal and occipital lobes inversely associated with BMI6). Morys et al. (2021) reported that BMI, WHR, and body fat percentage were associated with thinner temporal, entorhinal, orbitofrontal, and cingulate cortices, as well as thicker frontal, parietal, and occipital cortices.

Emerging neuroimaging studies have suggested the role of visceral fat in the association between obesity and brain structures in adults (Debette et al., 2010; Isaac et al., 2011; Widya et al., 2015; Zsido et al., 2019). Debette et al. (2010) demonstrated that visceral fat on computed tomography (CT) had the strongest association with reduced total brain volumes when compared with other obesity indices (BMI, waist circumference, wait-to-hip ratio, and subcutaneous fat), and the association was independent of BMI and insulin resistance. Widya et al. (2015) reported that increased visceral fat (but not subcutaneous fat) was associated with microstructural brain tissue damage in the elderly. Zsido et al. (2019) demonstrated that increased visceral fat was associated with accelerated brain aging (based on structural brain networks derived from gray matter volume, cortical thickness, and surface area) in adults including elderly participants. Isaac et al. (2011) analyzed the data of 184 healthy elderly individuals using voxelbased morphometry, and found that visceral fat was inversely associated with cortical thicknesses in several focal regions (e.g., pre-central, post-central, superior temporal, and inferior parietal cortices). Although the ROI-based approach (compared with voxel-based morphometry) can facilitate clinical interpretation by predefining brain regions, no study has investigated the associations between visceral fat (as well as subcutaneous fat) and ROI-based cortical thicknesses.

Hence, the present study aimed to explore brain regions associated with abdominal fat in the elderly, using the ROI-based analysis of brain magnetic resonance images.

\section{MATERIALS AND METHODS}

\section{Study Participants}

This study recruited $\geq 60$ year-old individuals (without selfreported history of dementia, movement disorders, or stroke) through local advertisements between December 2015 and September 2017 in Incheon, Republic of Korea, as part of the EPINEF study. The survey was conducted at Gachon University Gil Medical Center (Incheon, South Korea). Using a standardized survey protocol, a total of 322 participants completed questionnaires (regarding demographic characteristics, medical history, and lifestyle behaviors), anthropometric measurement (weight and height), blood sampling, abdominal fat CT scans, mini-mental state examination (MMSE), and brain 3T MRI scans. Two participants who were found to have brain tumors on brain MRI were excluded. After excluding individuals with missing values, 316 participants (129 men and 187 women) were included in the study. All individuals provided written informed consent. The study was approved by the Institutional Review Board of Gachon University Gil Medical Center (approval No. GDIRB2015-225).

\section{Acquisition of Abdominal Fat Areas}

All subjects underwent $10-\mathrm{mm}$-slice CT scans (SOMATOM Sensation 64; Siemens Healthcare, Forchheim, Germany) at the umbilical level. The average value of pixels within the range of -200 to -20 Hounsfield units was used for the measurement of abdominal fat areas (Jackson and Thomas, 2004). The total visceral fat area and the subcutaneous fat area (unit: $\mathrm{cm}^{2}$ ) were measured with a commercial software program (syngo Volume; Siemens Healthcare, Forchheim, Germany).

\section{Acquisition of Brain Imaging Markers}

Brain 3D-T1-magnetization-prepared rapid gradient-echo (MPRAGE) images were obtained with a Siemens 3T Verio MRI, using a standardized MRI protocol. The image parameters used for 3D T1-MP-RAGE were as follows: repetition time, 1,900 ms; echo time, $2.93 \mathrm{~ms}$; flip angle, $8^{\circ}$; pixel bandwidth, $170 \mathrm{~Hz} /$ pixel; matrix size, $256 \times 208$; field of view, $256 \mathrm{~mm}$; number of excitations, 1; total acquisition time, 4 min $10 \mathrm{~s}$; voxel size, $1.0 \times 1.0 \times 1.0 \mathrm{~mm}^{3}$.

ROI-based analyses of the brain images were performed using the standard FreeSurfer 6.0.0 pipeline ${ }^{1}$, which consists of subcortical segmentation (Fischl et al., 2002, 2004a); cortical surface reconstruction (Dale et al., 1999; Fischl et al., 1999); cortical thickness mapping (Fischl and Dale, 2000); surfacebased inter-subject alignment (Fischl et al., 1999); and cortical parcellation (Fischl et al., 2004b; Desikan et al., 2006). Using

\footnotetext{
${ }^{1}$ http://surfer.nmr.mgh.harvard.edu/
} 
these serial procedures, we obtained estimates of regional cortical thickness (frontal, temporal, parietal, occipital, cingulate, and insula) and subcortical gray matter volume (thalamus, caudate, putamen, pallidum, hippocampus, amygdala, and nucleus accumbens). Global cortical thickness was calculated by averaging the six cortical thicknesses.

\section{Covariates}

The questionnaire included educational years, history of disease (hypertension, diabetes mellitus, dyslipidemia, and angina or myocardial infarction), smoking status (never, former, or current smoker), and alcohol consumption (currently drinking or not). Measured weight and height were used to calculate BMI (unit: $\mathrm{kg} / \mathrm{m}^{2}$ ). At least $12-\mathrm{h}$ fasting blood samples were tested for blood glucose and total cholesterol levels, and apolipoprotein $\mathrm{E}$ (APOE) genotyping.

\section{Statistical Analysis}

To explore the non-linear relationship between abdominal fat area and cortical thickness, we used a generalized additive model (GAM), including visceral fat area as a spline variable and global cortical thickness as a dependent variable. In this analysis, we adjusted for age, sex, educational years, hypertension, diabetes, dyslipidemia, angina or myocardial infarction, smoking status, alcohol consumption, APOE status (presence/absence of $\varepsilon 4$ allele), BMI, fasting blood glucose level, total cholesterol level, and intracranial volume (ICV). The degrees of freedom for the spline variable were automatically selected using the generalized cross validation method. A two-sided $p<0.05$ from analysis of deviance for GAM was considered as having a significant non-linear relationship. There were significant nonlinear relationships of visceral fat area with global $(p=0.02)$, frontal $(p=0.02)$, temporal $(p=0.02)$, and parietal thicknesses $(p<0.001)$, with an inverted $U$ shape (Figure 1). Hence, we classified visceral fat area into quintiles (quintile 5 as the highest; quintile 3 as the reference group) and entered the quintiles into a generalized linear model (GLM). This approach was used for all the regional cortical thicknesses (though occipital and insular thicknesses did not exhibit significant non-linear relationships) with a view to presenting results in a consistent
A

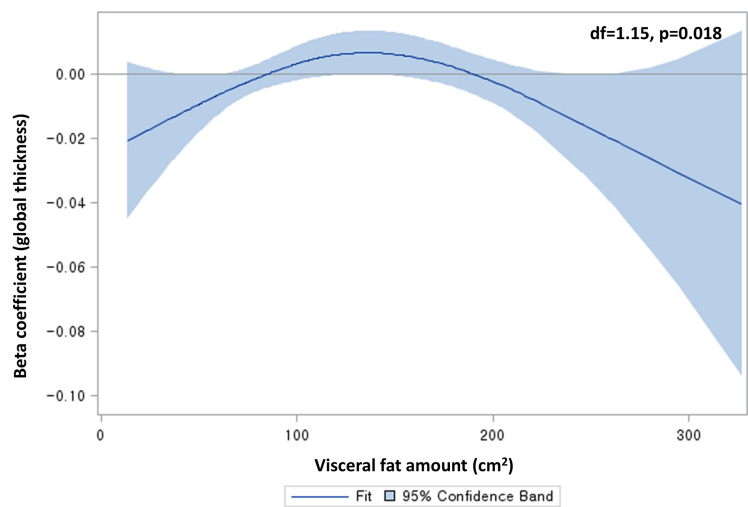

B

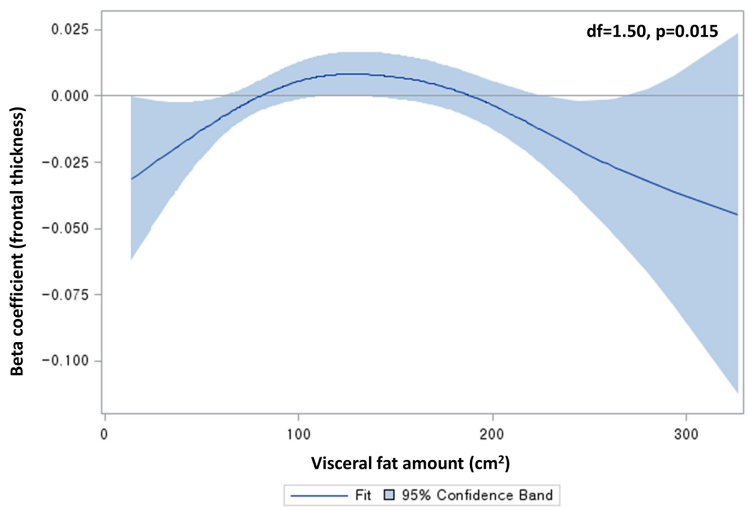

C

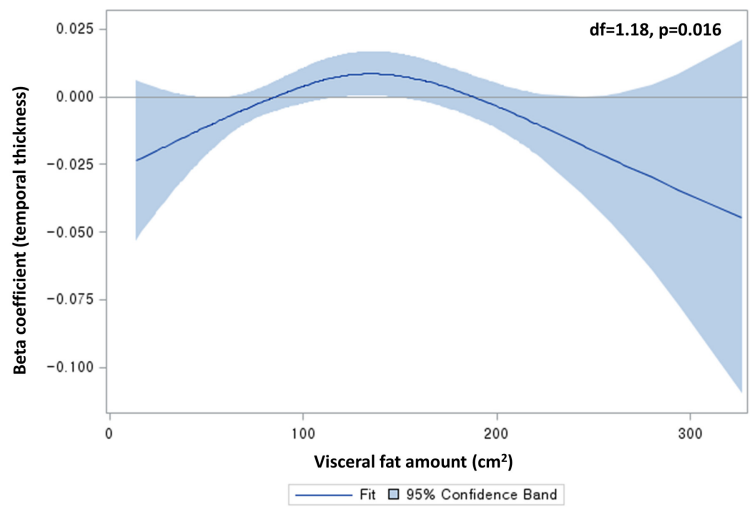

D

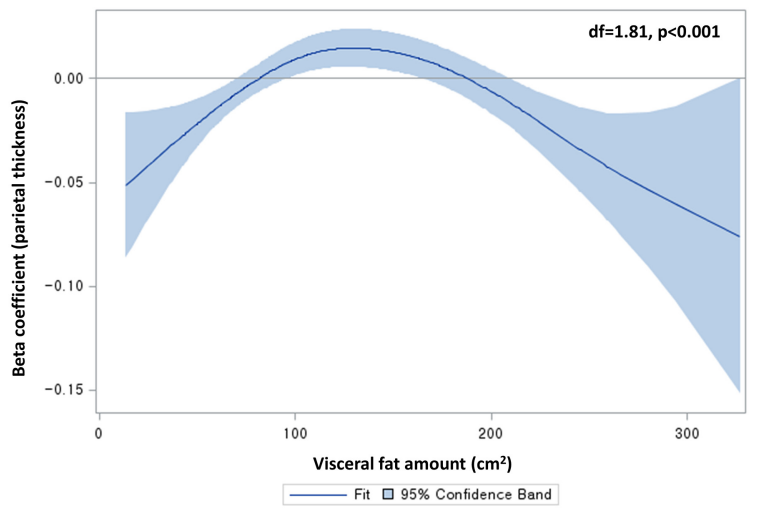

FIGURE 1 | Non-linear relationships of visceral fat area with (A) global, (B) frontal, (C) temporal, and (D) parietal cortical thicknesses. Df, degrees of freedom. Beta coefficients were from generalized additive models, adjusting for age, sex, educational years, hypertension, diabetes, dyslipidemia, angina or myocardial infarction, smoking status, alcohol consumption, apolipoprotein status, body mass index, fasting blood glucose level, total cholesterol level, and intracranial volume. Degrees of freedom were determined by the cross-validation method. 
manner. The same method was applied to subcutaneous fat area (albeit none of the non-linear relationships were significant) in order to enable a straightforward comparison with visceral fat. Given the absence of a significant non-linear relationship in the GAM analysis of subcortical volumes, the abdominal fat variables were entered as a continuous variable into the GLM for subcortical volumes. All GLM analyses were conducted after adjusting for the same covariates as the above GAM. Given possible sex differences in abdominal fat distribution as well as brain MRI markers (e.g., cortical thickness and volume) (Ritchie et al., 2018), sex-stratified analyses were additionally conducted. Significance of sex differences was tested using the method described by Altman and Bland and expressed as $\mathrm{p}$ for interaction (Altman and Bland, 2003). All analyses were corrected for multiple comparisons using the false discovery rate (FDR) method (Benjamini and Hochberg, 1995).

A post hoc analysis was conducted to examine the associations of other obesity indices (BMI and waist circumference) with brain cortical thickness. Quintiles of either BMI or waist circumference were entered into GLMs, with adjustment for the same covariates as the main analysis.

All statistical analyses were conducted using SAS version 9.4 (SAS Institute, Cary, NC, United States). Two-sided $p<0.05$ were considered statistically significant.

\section{RESULTS}

\section{Characteristics of Study Participants}

The mean [standard deviation (SD)] age of the study participants was 67.6 (5.0) (Table 1). The numbers of those with hypertension and dyslipidemia were 134 (42.4\%) and 104 (32.9\%), respectively. The mean (SD) areas of visceral fat and subcutaneous fat were $125.3(55.4) \mathrm{cm}^{2}$ and $167.5(65.7) \mathrm{cm}^{2}$, respectively. The mean (SD) MMSE score was 28.3 (1.9). The mean (SD) global thickness was $2.5(0.1) \mathrm{mm}$.

\section{Association Between Abdominal Fat Area and Cortical Thickness}

The quintile 3 group of visceral fat area had the greatest global cortical thickness (mean, $2.52 \mathrm{~mm}$; SD, $0.07 \mathrm{~mm}$ ), whereas the quintile 5 group had the smallest (mean, $2.48 \mathrm{~mm}$; SD, $0.08 \mathrm{~mm}$ ) (Table 2). In the GLM analysis of visceral fat (Table 3 ), the quintile 5 group (vs. the quintile 3 group) had significantly reduced cortical thicknesses in the global $[\beta=-0.04 \mathrm{~mm}$, standard error $(\mathrm{SE})=0.02 \mathrm{~mm}, p=0.004]$, parietal $(\beta=-0.04 \mathrm{~mm}, S E=0.02 \mathrm{~mm}, \quad p=0.01)$, temporal $(\beta=-0.05 \mathrm{~mm}, S E=0.02 \mathrm{~mm}, p=0.002)$, cingulate $(\beta=-0.06 \mathrm{~mm}, S E=0.02 \mathrm{~mm}, p=0.01)$, and insula lobes $(\beta=-0.06 \mathrm{~mm}, S E=0.03 \mathrm{~mm}, p=0.02)$. These associations remained significant after FDR correction. In men, the quintile 5 group (vs. the quintile 3 group) had significantly reduced global, temporal, and insular thicknesses, though these associations did not remain significant after FDR correction. In women, the quintiles 2, 4, and 5 groups had significantly reduced global cortical thicknesses, as compared with the quintile 3 group. The quintile 5 group (vs. the quintile 3 group) among women also
TABLE 1 | Characteristics of the study participants.

\begin{tabular}{|c|c|c|c|}
\hline & Total $(N=316)$ & Men $(N=129)$ & Women $(N=187)$ \\
\hline Age, mean (SD) & $67.6(5.0)$ & $68.9(4.9)$ & $66.7(5.0)$ \\
\hline $\begin{array}{l}\text { Educational years, } \\
\text { mean (SD) }\end{array}$ & $9.6(4.3)$ & $11.1(4.1)$ & $8.6(4.0)$ \\
\hline $\begin{array}{l}\text { Hypertension, N } \\
(\%)\end{array}$ & $134(42.4)$ & $60(46.5)$ & $74(39.6)$ \\
\hline $\begin{array}{l}\text { Diabetes mellitus, N } \\
(\%)\end{array}$ & $62(19.6)$ & $32(24.8)$ & $30(16.0)$ \\
\hline Dyslipidemia, N (\%) & $104(32.9)$ & $35(27.1)$ & 69 (36.9) \\
\hline $\begin{array}{l}\text { Angina or } \\
\text { myocardial } \\
\text { infarction, } N(\%)\end{array}$ & $37(11.7)$ & $19(14.7)$ & $18(9.6)$ \\
\hline \multicolumn{4}{|c|}{ Smoking status, N (\%) } \\
\hline Never smoker & $214(67.7)$ & $29(22.5)$ & 185 (98.9) \\
\hline Former smoker & $79(25.0)$ & $77(59.7)$ & $2(1.1)$ \\
\hline Current smoker & $23(7.3)$ & $23(17.8)$ & $0(0.0)$ \\
\hline $\begin{array}{l}\text { Alcohol drinking, N } \\
\text { (\%) }\end{array}$ & $113(35.8)$ & $75(58.1)$ & $38(20.3)$ \\
\hline $\begin{array}{l}\text { Body mass index, } \\
\text { mean (SD) }\end{array}$ & $24.7(3.1)$ & $24.8(2.6)$ & $24.7(3.4)$ \\
\hline \multicolumn{4}{|c|}{ Apolipoprotein status, N (\%) } \\
\hline $\begin{array}{l}\text { At least one } \varepsilon 4 \\
\text { allele }\end{array}$ & $56(17.7)$ & $26(20.2)$ & $30(16.0)$ \\
\hline No $\& 4$ allele & 260 (82.3) & $103(79.8)$ & $157(84.0)$ \\
\hline $\begin{array}{l}\text { Fasting blood } \\
\text { glucose, mean (SD) }\end{array}$ & $99.0(21.7)$ & $100.0(22.3)$ & $98.4(21.4)$ \\
\hline $\begin{array}{l}\text { Total cholesterol, } \\
\text { mean (SD) }\end{array}$ & $183.7(37.7)$ & $176.1(38.0)$ & $189.0(36.7)$ \\
\hline \multicolumn{4}{|c|}{ Abdominal fat $\left(\mathrm{cm}^{2}\right)$, mean (SD) } \\
\hline Visceral fat & $125.3(55.4)$ & $135.9(59.9)$ & $118.0(51.0)$ \\
\hline Subcutaneous fat & $167.5(65.7)$ & $130.1(46.8)$ & $193.3(64.5)$ \\
\hline $\begin{array}{l}\text { ICV }\left(\mathrm{mm}^{3}\right) \text {, mean } \\
\text { (SD) }\end{array}$ & $1,251,894(126,138)$ & $1,346,200(101,857)$ & ) $1,186,839(96,866)$ \\
\hline \multicolumn{4}{|c|}{ Cortical thickness (mm), mean (SD) } \\
\hline Global & $2.5(0.1)$ & $2.5(0.1)$ & $2.5(0.1)$ \\
\hline Frontal lobe & $2.5(0.1)$ & $2.5(0.1)$ & $2.6(0.1)$ \\
\hline Parietal lobe & $2.8(0.1)$ & $2.7(0.1)$ & $2.8(0.1)$ \\
\hline Temporal lobe & $2.3(0.1)$ & $2.2(0.1)$ & $2.3(0.1)$ \\
\hline Occipital lobe & $2.0(0.1)$ & $2.0(0.1)$ & $2.0(0.1)$ \\
\hline Cingulate & $2.6(0.1)$ & $2.5(0.1)$ & $2.6(0.1)$ \\
\hline Insula & $2.9(0.1)$ & $2.9(0.1)$ & $2.9(0.1)$ \\
\hline \multicolumn{4}{|c|}{ Subcortical volume $\left(\mathrm{mm}^{3}\right)$, mean (SD) } \\
\hline Thalamus & $6474.9(649.2)$ & 6691.9 (666.2) & $6325.2(594.2)$ \\
\hline Caudate & 3256.3 (477.7) & 3444.6 (476.3) & $3126.4(434.5)$ \\
\hline Putamen & $4461.1(520.1)$ & 4651.5 (534.4) & $4329.7(468.0)$ \\
\hline Pallidum & $1894.8(205.4)$ & 1960.8 (195.8) & 1849.3 (199.8) \\
\hline Amygdala & 1637.6 (193.7) & 1707.5 (187.4) & $1589.4(183.5)$ \\
\hline Hippocampus & 3903.3 (379.9) & 3993.8 (363.4) & 3840.9 (379.3) \\
\hline $\begin{array}{l}\text { Nucleus } \\
\text { accumbens }\end{array}$ & $427.4(75.0)$ & $447.1(78.8)$ & $413.8(69.3)$ \\
\hline
\end{tabular}

$S D$, standard deviation, $I C V$, intracranial volume.

had a significantly reduced parietal thickness $(\beta=-0.04 \mathrm{~mm}$, $S E=0.02 \mathrm{~mm}, p=0.04)$. After FDR correction, reduced global thicknesses in the quintiles 2 and 3 groups, a reduced frontal thickness in the quintile 4 group, a reduced parietal 
TABLE 2 | Global cortical thickness by quintiles of abdominal fat area.

\begin{tabular}{|c|c|c|c|c|c|c|c|}
\hline & \multirow[b]{2}{*}{$\mathbf{N}$} & \multicolumn{4}{|c|}{ Abdominal fat area } & \multicolumn{2}{|c|}{ Global cortical thickness } \\
\hline & & Mean & $S D$ & Minimum & Maximum & Mean & $S D$ \\
\hline \multicolumn{8}{|c|}{ Visceral fat } \\
\hline \multicolumn{8}{|c|}{ Total $(N=316)$} \\
\hline Quintile 1 & 63 & 54.68 & 15.51 & 13.66 & 74.13 & 2.50 & 0.08 \\
\hline Quintile 2 & 63 & 91.12 & 9.48 & 74.58 & 107.92 & 2.50 & 0.08 \\
\hline Quintile 3 & 64 & 120.08 & 7.96 & 108.22 & 135.14 & 2.52 & 0.07 \\
\hline Quintile 4 & 63 & 153.20 & 10.40 & 135.50 & 171.18 & 2.51 & 0.08 \\
\hline Quintile 5 & 63 & 207.75 & 33.47 & 171.24 & 326.45 & 2.48 & 0.08 \\
\hline \multicolumn{8}{|c|}{ Men $(N=129)$} \\
\hline Quintile 1 & 25 & 53.24 & 18.36 & 13.66 & 75.27 & 2.47 & 0.08 \\
\hline Quintile 2 & 26 & 98.14 & 13.70 & 75.81 & 117.24 & 2.50 & 0.08 \\
\hline Quintile 3 & 26 & 134.38 & 10.32 & 118.03 & 150.33 & 2.50 & 0.08 \\
\hline Quintile 4 & 26 & 170.17 & 9.72 & 152.63 & 187.48 & 2.47 & 0.07 \\
\hline Quintile 5 & 26 & 220.63 & 25.49 & 191.04 & 280.17 & 2.46 & 0.07 \\
\hline \multicolumn{8}{|c|}{ Women $(N=187)$} \\
\hline Quintile 1 & 37 & 55.19 & 13.42 & 16.87 & 73.47 & 2.52 & 0.07 \\
\hline Quintile 2 & 38 & 87.99 & 8.26 & 73.71 & 99.87 & 2.50 & 0.09 \\
\hline Quintile 3 & 37 & 112.48 & 6.75 & 100.05 & 125.28 & 2.55 & 0.06 \\
\hline Quintile 4 & 38 & 140.85 & 10.77 & 125.62 & 158.11 & 2.51 & 0.07 \\
\hline Quintile 5 & 37 & 193.86 & 39.00 & 159.1 & 326.45 & 2.52 & 0.08 \\
\hline \multicolumn{8}{|c|}{ Subcutaneous fat } \\
\hline \multicolumn{8}{|c|}{ Total $(N=316)$} \\
\hline Quintile 1 & 63 & 90.06 & 23.79 & 15.75 & 115.14 & 2.48 & 0.07 \\
\hline Quintile 2 & 63 & 127.14 & 7.41 & 115.51 & 140.91 & 2.49 & 0.09 \\
\hline Quintile 3 & 64 & 157.95 & 9.71 & 142.03 & 173.87 & 2.51 & 0.07 \\
\hline Quintile 4 & 63 & 194.44 & 13.05 & 174.85 & 220.18 & 2.50 & 0.07 \\
\hline Quintile 5 & 63 & 268.05 & 47.02 & 221.54 & 417.81 & 2.52 & 0.08 \\
\hline \multicolumn{8}{|c|}{ Men $(N=129)$} \\
\hline Quintile 1 & 25 & 69.99 & 21.65 & 15.75 & 92.57 & 2.46 & 0.07 \\
\hline Quintile 2 & 26 & 106.58 & 7.88 & 93.16 & 116.16 & 2.48 & 0.08 \\
\hline Quintile 3 & 26 & 125.29 & 4.80 & 116.23 & 133.09 & 2.47 & 0.10 \\
\hline Quintile 4 & 26 & 146.75 & 7.65 & 133.71 & 160.05 & 2.49 & 0.07 \\
\hline Quintile 5 & 26 & 199.35 & 33.93 & 163.84 & 306.01 & 2.48 & 0.06 \\
\hline \multicolumn{8}{|c|}{ Women $(N=187)$} \\
\hline Quintile 1 & 37 & 114.85 & 18.21 & 40.03 & 138.36 & 2.51 & 0.07 \\
\hline Quintile 2 & 38 & 155.22 & 9.93 & 139.16 & 170.92 & 2.52 & 0.07 \\
\hline Quintile 3 & 37 & 184.30 & 8.88 & 171.04 & 198.74 & 2.53 & 0.07 \\
\hline Quintile 4 & 38 & 222.01 & 12.63 & 199.73 & 242.21 & 2.51 & 0.08 \\
\hline Quintile 5 & 37 & 290.52 & 48.13 & 243.25 & 417.81 & 2.54 & 0.08 \\
\hline
\end{tabular}

SD, standard deviation.

thickness in the quintile 1 group, a reduced occipital thickness in the quintile 1 group, and a reduced cingulate thickness in the quintile 2 group remained significant. Regarding sex differences, the quintile 2 (vs. quintile 3) group had reduced cortical thicknesses among women but increased thicknesses among men in the global ( $\mathrm{p}$ for interaction $=0.024)$, frontal $(\mathrm{p}$ for interaction $=0.036)$, parietal $(\mathrm{p}$ for interaction $=0.007)$, and cingulate lobes $(\mathrm{p}$ for interaction $=0.021)$. Otherwise sex differences were not significant.

In the GLM analysis of subcutaneous fat (Supplementary Table 1), there were no significant differences in global cortical thickness across the quintile groups. The quintile 4 group had significantly reduced frontal $(\beta=-0.03 \mathrm{~mm}, S E=0.02 \mathrm{~mm}$, $p=0.03)$, temporal $(\beta=-0.03 \mathrm{~mm}, S E=0.02 \mathrm{~mm}, p=0.04)$, and occipital thicknesses $(\beta=-0.03 \mathrm{~mm}, S E=0.02 \mathrm{~mm}, p=0.03)$, as compared with the quintile 3 group. These associations did not remain significant after FDR correction. After stratification by sex, none of the associations remained significant.

\section{Association Between Abdominal Fat Area and Subcortical Volume}

In the GLM analysis of visceral fat area (Table 4), an increase in visceral fat area was significantly associated with reduced volumes of the pallium $\left(\beta=-0.66 \mathrm{~mm}^{3}, S E=0.25 \mathrm{~mm}^{3}\right.$, $p=0.01)$ and putamen $\left(\beta=-1.36 \mathrm{~mm}^{3}, S E=0.63 \mathrm{~mm}^{3}\right.$, $p=0.03)$. In men, the association between visceral fat area and the reduced volume of the pallidum was significant $\left(\beta=-0.55 \mathrm{~mm}^{3}\right.$, $\left.S E=0.25 \mathrm{~mm}^{3}, p=0.03\right)$. In women, the association between visceral fat area and the reduced volume of the putamen was significant $\left(\beta=-1.78 \mathrm{~mm}^{3}, S E=0.90 \mathrm{~mm}^{3}, p=0.05\right)$. None of the associations between subcutaneous fat area and subcortical 
TABLE 3 | Association between visceral fat area and cortical thickness.

\begin{tabular}{|c|c|c|c|c|c|c|c|c|c|c|c|}
\hline & & \multicolumn{3}{|c|}{ Total $(N=316)$} & \multicolumn{3}{|c|}{ Men $(N=129)$} & \multicolumn{3}{|c|}{ Women $(N=187)$} & \multirow[t]{2}{*}{$p$ for interaction ${ }^{\dagger}$} \\
\hline & & Beta & $S E$ & $p$ & Beta & $S E$ & $p$ & Beta & $S E$ & $p$ & \\
\hline \multirow[t]{4}{*}{ Global } & Quintile 1 vs. 3 & -0.008 & 0.015 & 0.61 & -0.022 & 0.024 & 0.35 & -0.022 & 0.02 & 0.27 & 1.00 \\
\hline & Quintile 2 vs. 3 & -0.01 & 0.014 & 0.45 & 0.017 & 0.023 & 0.45 & -0.049 & 0.018 & $0.009^{\star}$ & 0.024 \\
\hline & Quintile 4 vs. 3 & -0.011 & 0.014 & 0.42 & -0.035 & 0.023 & 0.13 & -0.05 & 0.018 & $0.007^{\star}$ & 0.61 \\
\hline & Quintile 5 vs. 3 & -0.043 & 0.015 & $0.004^{\star}$ & -0.05 & 0.024 & 0.042 & -0.04 & 0.019 & 0.038 & 0.74 \\
\hline \multirow[t]{4}{*}{ Frontal } & Quintile 1 vs. 3 & -0.019 & 0.017 & 0.27 & -0.014 & 0.025 & 0.58 & -0.035 & 0.022 & 0.12 & 0.53 \\
\hline & Quintile 2 vs. 3 & -0.007 & 0.015 & 0.63 & 0.027 & 0.024 & 0.28 & -0.04 & 0.021 & 0.055 & 0.036 \\
\hline & Quintile 4 vs. 3 & -0.013 & 0.015 & 0.40 & -0.019 & 0.024 & 0.44 & -0.059 & 0.021 & $0.004^{\star}$ & 0.21 \\
\hline & Quintile 5 vs. 3 & -0.028 & 0.016 & 0.082 & -0.014 & 0.026 & 0.58 & -0.035 & 0.021 & 0.11 & 0.53 \\
\hline \multirow[t]{4}{*}{ Parietal } & Quintile 1 vs. 3 & -0.031 & 0.017 & 0.069 & -0.03 & 0.029 & 0.29 & -0.053 & 0.021 & $0.011^{*}$ & 0.52 \\
\hline & Quintile 2 vs. 3 & -0.009 & 0.016 & 0.58 & 0.045 & 0.028 & 0.11 & -0.047 & 0.019 & 0.017 & 0.007 \\
\hline & Quintile 4 vs. 3 & -0.012 & 0.015 & 0.43 & 0.006 & 0.028 & 0.82 & -0.045 & 0.019 & 0.021 & 0.13 \\
\hline & Quintile 5 vs. 3 & -0.041 & 0.016 & $0.013^{*}$ & -0.038 & 0.03 & 0.20 & -0.042 & 0.02 & 0.039 & 0.91 \\
\hline \multirow[t]{4}{*}{ Temporal } & Quintile 1 vs. 3 & -0.019 & 0.018 & 0.28 & -0.041 & 0.026 & 0.13 & -0.014 & 0.023 & 0.55 & 0.44 \\
\hline & Quintile 2 vs. 3 & -0.024 & 0.016 & 0.14 & -0.008 & 0.025 & 0.74 & -0.049 & 0.022 & 0.026 & 0.22 \\
\hline & Quintile 4 vs. 3 & -0.02 & 0.016 & 0.22 & -0.06 & 0.025 & 0.019 & -0.043 & 0.022 & 0.046 & 0.61 \\
\hline & Quintile 5 vs. 3 & -0.054 & 0.017 & $0.002^{\star}$ & -0.062 & 0.027 & 0.026 & -0.043 & 0.022 & 0.056 & 0.59 \\
\hline \multirow[t]{4}{*}{ Occipital } & Quintile 1 vs. 3 & -0.037 & 0.017 & $0.03^{\star}$ & -0.02 & 0.028 & 0.47 & -0.065 & 0.021 & $0.003^{\star}$ & 0.20 \\
\hline & Quintile 2 vs. 3 & -0.016 & 0.016 & 0.31 & 0.021 & 0.027 & 0.43 & -0.034 & 0.02 & 0.094 & 0.10 \\
\hline & Quintile 4 vs. 3 & -0.014 & 0.015 & 0.35 & -0.004 & 0.027 & 0.87 & -0.027 & 0.02 & 0.17 & 0.49 \\
\hline & Quintile 5 vs. 3 & -0.016 & 0.016 & 0.32 & -0.018 & 0.028 & 0.54 & -0.016 & 0.02 & 0.44 & 0.95 \\
\hline \multirow[t]{4}{*}{ Cingulate } & Quintile 1 vs. 3 & 0.02 & 0.023 & 0.37 & -0.007 & 0.035 & 0.83 & 0.013 & 0.028 & 0.65 & 0.66 \\
\hline & Quintile 2 vs. 3 & -0.02 & 0.02 & 0.32 & 0.022 & 0.034 & 0.52 & -0.078 & 0.027 & $0.004^{\star}$ & 0.021 \\
\hline & Quintile 4 vs. 3 & -0.004 & 0.02 & 0.85 & -0.034 & 0.034 & 0.31 & -0.056 & 0.026 & 0.034 & 0.61 \\
\hline & Quintile 5 vs. 3 & -0.06 & 0.022 & $0.006^{\star}$ & -0.056 & 0.036 & 0.12 & -0.05 & 0.027 & 0.068 & 0.89 \\
\hline \multirow[t]{4}{*}{ Insula } & Quintile 1 vs. 3 & 0.039 & 0.026 & 0.14 & -0.021 & 0.039 & 0.60 & 0.024 & 0.034 & 0.48 & 0.38 \\
\hline & Quintile 2 vs. 3 & 0.013 & 0.024 & 0.59 & -0.003 & 0.037 & 0.94 & -0.044 & 0.032 & 0.17 & 0.40 \\
\hline & Quintile 4 vs. 3 & -0.004 & 0.024 & 0.85 & -0.095 & 0.037 & 0.012 & -0.069 & 0.032 & 0.031 & 0.60 \\
\hline & Quintile 5 vs. 3 & -0.059 & 0.025 & $0.019^{\star}$ & -0.112 & 0.04 & 0.006 & -0.052 & 0.033 & 0.11 & 0.25 \\
\hline
\end{tabular}

SE, standard error.

Beta coefficients were from generalized linear models, adjusting for age, sex, educational years, hypertension, diabetes, dyslipidemia, angina or myocardial infarction, smoking status, alcohol consumption, apolipoprotein status, body mass index, fasting blood glucose level, total cholesterol level, and intracranial volume. The quintile 3

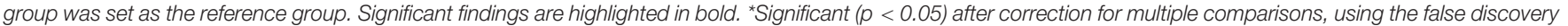
rate method.

†Significance of sex differences.

volumes were significant. After FDR correction, none of the associations remained significant.

\section{Post hoc Analyses}

The quintile 1 group of BMI had significantly reduced global $(\beta=-0.03 \mathrm{~mm}, S E=0.01 \mathrm{~mm}, p=0.027)$, parietal $(\beta=-0.06 \mathrm{~mm}, \quad S E=0.02 \mathrm{~mm}, \quad p<0.001)$, temporal $(\beta=-0.05 \mathrm{~mm}, S E=0.02 \mathrm{~mm}, p=0.004)$, and occipital thicknesses $(\beta=-0.04 \mathrm{~mm}, S E=0.02 \mathrm{~mm}, p=0.007)$, as compared with the quintile 3 group. Other findings are presented in Supplementary Table 2. In the analyses of waist circumference, none of the associations were significant except a reduced parietal thickness in the quintile 1 group $(\beta=-0.04 \mathrm{~mm}$, $S E=0.02 \mathrm{~mm}, p=0.015)$. Other findings are presented in Supplementary Table 3.

\section{DISCUSSION}

The present study is the first to investigate the associations of visceral and subcutaneous fat area with ROI-based cortical thicknesses and subcortical volumes in elderly individuals without dementia. This neuroimaging study involved a relatively large sample size $(n=316)$ and adjusted for a range of covariates including well-known metabolic risk factors, as well as the apolipoprotein $\varepsilon 4$ allele-the major genetic risk factor for Alzheimer's disease. The main finding was that individuals with the highest level of visceral fat area had significantly reduced cortical thicknesses in the global, parietal, temporal, cingulate, and insular lobes, as compared with those with the middle level of visceral fat area. These associations did not significantly differ by sex. By contrast, none of the regional cortical thicknesses significantly differed between individuals with the highest level and those with the middle level of subcutaneous fat area.

In recent decades, there has been debate surrounding the effect of high BMI on dementia risk. The largest cohort study on this topic (of two million individuals) demonstrated a protective effect of higher BMI (Qizilbash et al., 2015), while a meta-analysis of four studies showed a harmful effect of obesity (Pedditzi et al., 2016). Another cohort study of 1.3 million individuals suggested a harmful effect of higher BMI on dementia risk over $>20$ years of follow-up, as well as a protective effect of higher BMI over $\leq 20$ years of follow-up, 
TABLE 4 | Association between abdominal fat area and subcortical volume.

\begin{tabular}{|c|c|c|c|c|c|c|c|c|c|c|}
\hline & \multicolumn{3}{|c|}{ Total $(N=316)$} & \multicolumn{3}{|c|}{ Men $(N=129)$} & \multicolumn{3}{|c|}{ Women $(N=187)$} & \multirow[t]{2}{*}{$\boldsymbol{p}$ for interaction ${ }^{\dagger}$} \\
\hline & Beta & $S E$ & $p$ & Beta & $S E$ & $p$ & Beta & $S E$ & $p$ & \\
\hline \multicolumn{11}{|l|}{ Visceral fat } \\
\hline Thalamus & -0.383 & 0.643 & 0.55 & 0.2 & 0.988 & 0.84 & -0.935 & 0.913 & 0.31 & 0.40 \\
\hline Caudate & -0.928 & 0.609 & 0.13 & -0.742 & 0.951 & 0.44 & -1.144 & 0.877 & 0.19 & 0.76 \\
\hline Pallidum & -0.664 & 0.25 & 0.008 & -0.79 & 0.348 & 0.025 & -0.584 & 0.385 & 0.13 & 0.69 \\
\hline Putamen & -1.355 & 0.627 & 0.031 & -1.247 & 0.983 & 0.21 & -1.781 & 0.895 & 0.048 & 0.69 \\
\hline Amygdala & 0.203 & 0.224 & 0.37 & 0.226 & 0.346 & 0.52 & 0.164 & 0.33 & 0.62 & 0.90 \\
\hline Hippocampus & -0.286 & 0.428 & 0.50 & -0.129 & 0.626 & 0.84 & -0.597 & 0.641 & 0.35 & 0.60 \\
\hline Nucleus accumbens & -0.132 & 0.093 & 0.16 & -0.1 & 0.135 & 0.46 & -0.186 & 0.136 & 0.17 & 0.65 \\
\hline \multicolumn{11}{|l|}{ Subcutaneous fat } \\
\hline Thalamus & -0.217 & 0.624 & 0.73 & -1.258 & 1.258 & 0.32 & 0.351 & 0.72 & 0.63 & 0.27 \\
\hline Caudate & -0.602 & 0.592 & 0.31 & -1.645 & 1.209 & 0.18 & -0.445 & 0.692 & 0.52 & 0.39 \\
\hline Pallidum & -0.008 & 0.246 & 0.97 & -0.026 & 0.455 & 0.95 & 0.051 & 0.305 & 0.87 & 0.89 \\
\hline Putamen & 0.307 & 0.612 & 0.62 & 0.877 & 1.263 & 0.49 & 0.134 & 0.712 & 0.85 & 0.61 \\
\hline Amygdala & 0.047 & 0.218 & 0.83 & 0.278 & 0.442 & 0.53 & -0.077 & 0.26 & 0.77 & 0.49 \\
\hline Hippocampus & -0.148 & 0.416 & 0.72 & 0.722 & 0.797 & 0.37 & -0.395 & 0.504 & 0.44 & 0.24 \\
\hline Nucleus accumbens & -0.157 & 0.09 & 0.082 & -0.091 & 0.173 & 0.60 & -0.165 & 0.107 & 0.13 & 0.72 \\
\hline
\end{tabular}

SE, standard error.

Beta coefficients were from generalized linear models, adjusting for age, sex, educational years, hypertension, diabetes, dyslipidemia, angina or myocardial infarction,

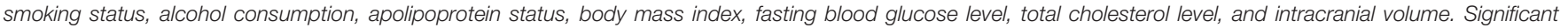
findings are highlighted in bold. None of the associations remained significant after correction for multiple comparisons using the false discovery rate method.

†Significance of sex differences.

possibly due to reverse causation (Kivimaki et al., 2018). Another meta-analysis of 10 prospective cohort studies demonstrated a significant U-shaped association between BMI and dementia risk, indicating that both underweight individuals and overweight individuals are at risk of dementia (Beydoun et al., 2008). This controversial relationship between BMI and dementia and its underlying mechanisms can be, at least in part, scrutinized by using more intricate biomarkers in imaging studies. An MRI analysis of 1,777 cognitively healthy individuals found a significant inverted U-shaped relationship between central obesity (WHR as a proxy) and global cortical thickness (Kim et al., 2015). In line with this, we found a significant inverted $\mathrm{U}$-shaped relationship between visceral fat area on CT and global cortical thickness. It is noteworthy that, when compared with the middle quintile group of visceral fat area, global cortical thinning was significant in the highest quintile group, but not in the lowest quintile group. This highlights a harmful effect of high visceral fat on brain gray matter, as the previous neuroimaging studies have suggested (Debette et al., 2010; Isaac et al., 2011; Widya et al., 2015; Zsido et al., 2019). Taken together, it is possible that high visceral fat leads to cortical thinning and, hence, contribute to the increased risk of dementia in overweight or obese individuals. Further, in concordance with the previous study using voxel-based morphometry (Isaac et al., 2011), the present study demonstrated that the highest level of visceral fat was significantly associated with reduced thicknesses in association cortices (critical for integrating sensory inputs) such as the temporal and parietal lobes. A similar pattern was observed in the cingulate cortex in the present study. These affected brain cortices correspond to the sites that show atrophy in the early stage of mild cognitive impairment (McDonald et al., 2009). Hence, it is reasonable to suggest that individuals with high visceral fat may initially develop preclinical cortical thinning in the temporal, parietal, and cingulate lobes, followed by clinical outcomes such as mild cognitive impairment.

Given possible correlations between visceral fat area and other obesity indices (e.g., BMI and waist circumference), it is possible that the association between visceral fat and cortical thickness was driven by the impact of other obesity indices. In the present study, there was a significant correlation between BMI and visceral fat area (Supplementary Figure 1), and cortical thinning associated with the highest visceral fat group was found significant in the temporal, parietal, cingulate, and insular lobes, after adjusting for a range of covariates including BMI. Notably, a significant decline in cortical thickness was mainly observed in the highest quintile groups of visceral fat area, whereas cortical thinning was significant only in the lowest quintile groups of BMI or waist circumference (Supplementary Tables 2, 3). This suggests that visceral fat area, compared with BMI and waist circumference, might be a better indicator of obesity-induced cortical thinning. In line with this, an analysis of the Framingham Offspring cohort demonstrated that visceral fat was more strongly associated with decreased cerebral volumes compared with BMI, waist circumference, or subcutaneous fat (Debette et al., 2010). Furthermore, the relationship between visceral fat and cortical thinning is supported by animal studies, demonstrating plausible biological mechanisms such as microglial activation, upregulated pro-inflammatory cytokines in the brain, and increased blood-brain barrier permeability via visceral fat inflammation (Shin et al., 2015; Guo et al., 2020). Epidemiological evidence also suggests that visceral fat deposition may induce systemic inflammation and, in turn, cerebral small-vessel disease (e.g., white matter hyperintensities), which is related to reduced cortical thickness (Lampe et al., 2019; Morys et al., 2021). 
There are several limitations to be noted. First, we cannot establish temporality between a high level of visceral fat and cortical thinning due to the cross-sectional nature of the study. Longitudinal investigations are warranted to clarify the temporal relationship between high visceral fat area and cortical thinning. Second, our findings might not be generalizable to other ethnic populations. In particular, the inverted U-shaped relationship between visceral fat area and cortical thickness in our samples might not be present in Western populations, though a meta-analysis including 10 prospective studies demonstrated a U-shaped relationship between BMI and risk of dementia (Beydoun et al., 2008). Last, we included dementia-free individuals based on self-reported information, and this approach might not have captured variation in cognitive health (including subthreshold cognitive impairment). Besides, the MMSE may not be sensitive to mild cognitive impairment (Mitchell, 2009; Yim et al., 2021). Future studies are warranted to assess cognitive health with sufficient granularity to elucidate fat-related cortical thinning.

In conclusion, a high level of visceral fat was significantly associated with a reduced global cortical thickness in the brains of elderly individuals without dementia, movement disorders, or stroke. Cortical thinning associated with the highest level of visceral fat area was significant in the parietal, temporal, cingulate, and insular lobes, whereas cortical thinning associated with the highest level of subcutaneous fat area was not significant in any of the studied lobes. These findings support the role of visceral fat in obesity-induced neurodegeneration.

\section{DATA AVAILABILITY STATEMENT}

The original contributions presented in the study are included in the article/Supplementary Material, further inquiries can be directed to the corresponding author/s.

\section{REFERENCES}

Altman, D. G., and Bland, J. M. (2003). Interaction revisited: the difference between two estimates. BMJ 326:219. doi: 10.1136/bmj.326.7382.219

Bell, J. A., Kivimaki, M., and Hamer, M. (2014). Metabolically healthy obesity and risk of incident type 2 diabetes: a meta-analysis of prospective cohort studies. Obes. Rev. 15, 504-515. doi: 10.1111/obr.12157

Benjamini, Y., and Hochberg, Y. (1995). Controlling the false discovery rate: a practical and powerful approach to multiple testing. J. Royal Stat. Soc. Ser. B Methodol. 57, 289-300.

Beydoun, M. A., Beydoun, H. A., and Wang, Y. (2008). Obesity and central obesity as risk factors for incident dementia and its subtypes: a systematic review and meta-analysis. Obes. Rev. 9, 204-218. doi: 10.1111/j.1467-789X.2008.00 473.x

Bogers, R. P., Bemelmans, W. J., Hoogenveen, R. T., Boshuizen, H. C., Woodward, M., Knekt, P., et al. (2007). Association of overweight with increased risk of coronary heart disease partly independent of blood pressure and cholesterol levels: a meta-analysis of 21 cohort studies including more than 300000 persons. Arch. Intern. Med. 167, 1720-1728. doi: 10.1001/archinte.167. 16.1720

Burggren, A. C., Zeineh, M. M., Ekstrom, A. D., Braskie, M. N., Thompson, P. M., Small, G. W., et al. (2008). Reduced cortical thickness in hippocampal subregions among cognitively normal apolipoprotein E e4 carriers. Neuroimage 41, 1177-1183. doi: 10.1016/j.neuroimage.2008.03.039

\section{ETHICS STATEMENT}

The study was approved by the Institutional Review Board of Gachon University Gil Medical Center (approval No. GDIRB2015-225). The patients/participants provided their written informed consent to participate in this study.

\section{AUTHOR CONTRIBUTIONS}

JC, CK, and YN designed the study. JC and YN contributed to the data collection and drafted the manuscript. JC analyzed the data. SS, W-RK, and CK provided significant intellectual input and a critical review of the manuscript. All authors approved the final version of the manuscript.

\section{FUNDING}

This research was supported by grants from the Korea Health Technology R\&D Project through the Korea Health Industry Development Institute (KHIDI), funded by the Ministry of Health and Welfare, South Korea (Grant Nos. HI18C1629 and HI14C1135); Brain Research Program of the National Research Foundation (NRF) funded by the Korean Government (MSIT) (Grant No. 2018M3C7A1056889); and Research Program funded by the Korea Centers for Disease Control and Prevention (2020ER6706-00).

\section{SUPPLEMENTARY MATERIAL}

The Supplementary Material for this article can be found online at: https://www.frontiersin.org/articles/10.3389/fnagi. 2021.694629/full\#supplementary-material

Dale, A. M., Fischl, B., and Sereno, M. I. (1999). Cortical surface-based analysis. I. Segmentation and surface reconstruction. Neuroimage 9, 179-194. doi: 10.1006/ nimg.1998.0395

Debette, S., Beiser, A., Hoffmann, U., Decarli, C., O’donnell, C. J., Massaro, J. M., et al. (2010). Visceral fat is associated with lower brain volume in healthy middle-aged adults. Ann. Neurol. 68, 136-144. doi: 10.1002/ana. 22062

Dekkers, I. A., Jansen, P. R., and Lamb, H. J. (2019). Obesity, brain volume, and white matter microstructure at MRI: a cross-sectional UK biobank study. Radiology 291, 763-771. doi: 10.1148/radiol.2019181012

Desikan, R. S., Segonne, F., Fischl, B., Quinn, B. T., Dickerson, B. C., Blacker, D., et al. (2006). An automated labeling system for subdividing the human cerebral cortex on MRI scans into gyral based regions of interest. Neuroimage 31, 968-980. doi: 10.1016/j.neuroimage.2006.01.021

Fischl, B., and Dale, A. M. (2000). Measuring the thickness of the human cerebral cortex from magnetic resonance images. Proc. Natl. Acad. Sci. U.S.A. 97, 11050-11055. doi: 10.1073/pnas.200033797

Fischl, B., Salat, D. H., Busa, E., Albert, M., Dieterich, M., Haselgrove, C., et al. (2002). Whole brain segmentation: automated labeling of neuroanatomical structures in the human brain. Neuron 33, 341-355.

Fischl, B., Salat, D. H., Van Der Kouwe, A. J., Makris, N., Segonne, F., Quinn, B. T., et al. (2004a). Sequence-independent segmentation of magnetic resonance images. Neuroimage 23(Suppl. 1), S69-S84. doi: 10.1016/j.neuroimage.2004.07. 016 
Fischl, B., Van Der Kouwe, A., Destrieux, C., Halgren, E., Segonne, F., Salat, D. H., et al. (2004b). Automatically parcellating the human cerebral cortex. Cereb Cortex 14, 11-22. doi: 10.1093/cercor/bhg087

Fischl, B., Sereno, M. I., and Dale, A. M. (1999). Cortical surface-based analysis. II: inflation, flattening, and a surface-based coordinate system. Neuroimage 9, 195-207. doi: 10.1006/nimg.1998.0396

Gunstad, J., Paul, R. H., Cohen, R. A., Tate, D. F., Spitznagel, M. B., Grieve, S., et al. (2008). Relationship between body mass index and brain volume in healthy adults. Int. J. Neurosci. 118, 1582-1593. doi: 10.1080/00207450701392282

Guo, D.-H., Yamamoto, M., Hernandez, C. M., Khodadadi, H., Baban, B., and Stranahan, A. M. (2020). Visceral adipose NLRP3 impairs cognition in obesity via IL-1R1 on CX3CR1+ cells. J. Clin. Investigation 130, 1961-1976. doi: 10. 1172/JCI126078

Hamer, M., and Batty, G. D. (2019). Association of body mass index and waistto-hip ratio with brain structure: UK biobank study. Neurology 92, e594-e600. doi: 10.1212/WNL.0000000000006879

Isaac, V., Sim, S., Zheng, H., Zagorodnov, V., Tai, E. S., and Chee, M. (2011). Adverse associations between visceral adiposity, brain structure, and cognitive performance in healthy elderly. Front. Aging Neurosci. 3:12. doi: 10.3389/fnagi. 2011.00012

Jackson, S., and Thomas, R. (eds). (2004). "Computed tomography (CT): clinical applications of CT," in Cross-Sectional Imaging Made Easy, (Oxford: Churchill Livingstone).

Kim, H. J., Kim, C., Jeon, S., Kang, M., Kim, Y. J., Lee, J. M., et al. (2015). Association of body fat percentage and waist-hip ratio with brain cortical thickness: a study among 1777 cognitively normal subjects. Alzheimer Dis. Assoc. Disord. 29, 279-286. doi: 10.1097/WAD.00000000000 00079

Kivimaki, M., Luukkonen, R., Batty, G. D., Ferrie, J. E., Pentti, J., Nyberg, S. T., et al. (2018). Body mass index and risk of dementia: analysis of individuallevel data from 1.3 million individuals. Alzheimers Dement 14, 601-609. doi: 10.1016/j.jalz.2017.09.016

Kurth, F., Levitt, J. G., Phillips, O. R., Luders, E., Woods, R. P., Mazziotta, J. C., et al. (2013). Relationships between gray matter, body mass index, and waist circumference in healthy adults. Hum. Brain Mapp. 34, 1737-1746. doi: 10. 1002/hbm. 22021

Lampe, L., Zhang, R., Beyer, F., Huhn, S., Kharabian Masouleh, S., Preusser, S., et al. (2019). Visceral obesity relates to deep white matter hyperintensities via inflammation. Ann. Neurol. 85, 194-203. doi: 10.1002/ana.2 5396

McDonald, C. R., Mcevoy, L. K., Gharapetian, L., Fennema-Notestine, C., Hagler, D. J. Jr., Holland, D., et al. (2009). Regional rates of neocortical atrophy from normal aging to early Alzheimer disease. Neurology 73, 457-465. doi: 10.1212/ WNL.0b013e3181b16431

Medic, N., Ziauddeen, H., Ersche, K. D., Farooqi, I. S., Bullmore, E. T., Nathan, P. J., et al. (2016). Increased body mass index is associated with specific regional alterations in brain structure. Int. J. Obes (Lond) 40, 1177-1182. doi: 10.1038/ ijo. 2016.42

Mitchell, A. J. (2009). A meta-analysis of the accuracy of the mini-mental state examination in the detection of dementia and mild cognitive impairment. J. Psychiatr. Res. 43, 411-431. doi: 10.1016/j.jpsychires.2008.04.014

Morys, F., Dadar, M., and Dagher, A. (2021). Association between mid-life obesity, its metabolic consequences, cerebrovascular disease and cognitive decline. J. Clin. Endocrinol. Metab. 2:dgab135. doi: 10.1210/clinem/dgab135
Pedditzi, E., Peters, R., and Beckett, N. (2016). The risk of overweight/obesity in mid-life and late life for the development of dementia: a systematic review and meta-analysis of longitudinal studies. Age Ageing 45, 14-21. doi: 10.1093/ ageing/afv151

Qizilbash, N., Gregson, J., Johnson, M. E., Pearce, N., Douglas, I., Wing, K., et al. (2015). BMI and risk of dementia in two million people over two decades: a retrospective cohort study. Lancet Diabetes Endocrinol. 3, 431-436. doi: 10. 1016/S2213-8587(15)00033-9

Raji, C. A., Ho, A. J., Parikshak, N. N., Becker, J. T., Lopez, O. L., Kuller, L. H., et al. (2010). Brain structure and obesity. Hum. Brain Mapp. 31, 353-364. doi: 10.1002/hbm.20870

Renehan, A. G., Tyson, M., Egger, M., Heller, R. F., and Zwahlen, M. (2008). Bodymass index and incidence of cancer: a systematic review and meta-analysis of prospective observational studies. Lancet 371, 569-578. doi: 10.1016/S01406736(08)60269-X

Ritchie, S. J., Cox, S. R., Shen, X., Lombardo, M. V., Reus, L. M., Alloza, C., et al. (2018). Sex differences in the adult human brain: evidence from $5216 \mathrm{UK}$ biobank participants. Cereb Cortex 28, 2959-2975. doi: 10.1093/cercor/bhy109

Shin, J. A., Jeong, S. I., Kim, M., Yoon, J. C., Kim, H. S., and Park, E. M. (2015). Visceral adipose tissue inflammation is associated with age-related brain changes and ischemic brain damage in aged mice. Brain Behav. Immun. 50, 221-231. doi: 10.1016/j.bbi.2015.07.008

Taki, Y., Kinomura, S., Sato, K., Inoue, K., Goto, R., Okada, K., et al. (2008). Relationship between body mass index and gray matter volume in 1,428 healthy individuals. Obesity (Silver Spring) 16, 119-124. doi: 10.1038/oby.2007.4

Thambisetty, M., Wan, J., Carass, A., An, Y., Prince, J. L., and Resnick, S. M. (2010). Longitudinal changes in cortical thickness associated with normal aging. Neuroimage 52, 1215-1223. doi: 10.1016/j.neuroimage.2010.04.258

Widya, R. L., Kroft, L. J. M., Altmann-Schneider, I., Van Den Berg-Huysmans, A. A., Van Der Bijl, N., De Roos, A., et al. (2015). Visceral adipose tissue is associated with microstructural brain tissue damage. Obesity 23, 1092-1096. doi: $10.1002 /$ oby. 21048

Yim, Y., Lee, J. Y., Oh, S. W., Chung, M. S., Park, J. E., Moon, Y., et al. (2021). Comparison of automated brain volume measures by neuroquant vs. Freesurfer in patients with mild cognitive impairment: effect of slice thickness. Yonsei Med. J. 62, 255-261. doi: 10.3349/ymj.2021.62.3.255

Yokum, S., Ng, J., and Stice, E. (2012). Relation of regional gray and white matter volumes to current BMI and future increases in BMI: a prospective MRI study. Int. J. Obes (Lond) 36, 656-664. doi: 10.1038/ijo.2011.175

Zsido, R. G., Heinrich, M., Slavich, G. M., Beyer, F., Kharabian Masouleh, S., Kratzsch, J., et al. (2019). Association of estradiol and visceral fat with structural brain networks and memory performance in adults. JAMA Netw. Open 2:e196126. doi: 10.1001/jamanetworkopen.2019.6126

Conflict of Interest: The authors declare that the research was conducted in the absence of any commercial or financial relationships that could be construed as a potential conflict of interest.

Copyright (c) 2021 Cho, Seo, Kim, Kim and Noh. This is an open-access article distributed under the terms of the Creative Commons Attribution License (CC BY). The use, distribution or reproduction in other forums is permitted, provided the original author(s) and the copyright owner(s) are credited and that the original publication in this journal is cited, in accordance with accepted academic practice. No use, distribution or reproduction is permitted which does not comply with these terms. 